\title{
Novel Biallelic NSUN3 Variants Cause Early-Onset Mitochondrial Encephalomyopathy and Seizures
}

\author{
Arumugam Paramasivam ${ }^{1,2} \cdot$ Angamuthu K. Meena $^{3} \cdot$ Challa Venkatapathi $^{1} \cdot$ Robert D.S. Pitceathly $^{4}$ (D) \\ Kumarasamy Thangaraj ${ }^{1}$ (D)
}

Received: 12 August 2019 / Accepted: 14 May 2020 / Published online: 2 June 2020

(C) The Author(s) 2020, corrected publication July 2020

\begin{abstract}
Epitranscriptomic systems enable post-transcriptional modifications of cellular RNA that are essential for regulating gene expression. Of the $\sim 170$ known RNA chemical modifications, methylation is among the most common. Loss of function mutations in NSUN3, encoding the 5-methylcytosine $(\mathrm{m} 5 \mathrm{C})$ methyltransferase NSun3, have been linked to multisystem mitochondrial disease associated with combined oxidative phosphorylation deficiency. Here, we report a patient with early-onset mitochondrial encephalomyopathy and seizures in whom the novel biallelic NSUN3 missense variants c.421G $>C$ (p.A141P) and c.454T>A (p.C152S) were detected. Segregation studies and in silico functional analysis confirmed the likely pathogenic effects of both variants. These findings expand the molecular and phenotypic spectrum of NSUN3-related mitochondrial disease.
\end{abstract}

Keywords Epitranscriptomics $\cdot$ Mitochondrial disorders $\cdot$ mtDNA $\cdot$ NSUN3 $\cdot$ Encephalomyopathy $\cdot$ Seizures

\section{Introduction}

Epitranscriptomic systems are necessary for the posttranscriptional modification of cellular RNA, RNA splicing and protein translation (Hsu et al. 2017; Rozov et al. 2016). Of the $\sim 170$ RNA chemical modifications reported, methylation is among the most common (Machnicka et al. 2013), with more than 90 reactions involving tRNA molecules (Boccaletto et al. 2018; Hussain et al. 2016). Numerous RNA modification enzymes and catalytic RNA-protein complexes are necessary for the post-transcriptional modification

Robert D.S. Pitceathly and Kumarasamy Thangaraj are joint senior authors for this paper.

Kumarasamy Thangaraj

thangs@ccmb.res.in

1 CSIR-Centre for Cellular and Molecular Biology, Hyderabad, India

2 BRULAC-DRC, Saveetha Dental College and Hospital, Saveetha Institute of Medical and Technical Sciences, Saveetha University, Chennai, India

3 Department of Neurology, Nizam's Institute of Medical Sciences, Hyderabad, India

4 Department of Neuromuscular Diseases, UCL Queen Square Institute of Neurology and The National Hospital for Neurology and Neurosurgery, London, United Kingdom events (Rozov et al. 2016), although many remain poorly characterised.

Human diseases linked to defects in these pathways emphasise the important role of epitranscriptomics in gene expression (Hsu et al. 2017). One recent example involves the 5methylcytosine (m5C) methyltransferase NSun3, encoded by NOP2/Sun RNA methyltransferase 3 (NSUN3). Loss of function mutations in NSUN3 cause multisystem mitochondrial disease associated with a combined oxidative phosphorylation (OXPHOS) deficiency (Haag et al. 2016), highlighting the importance of NSun3 in mitochondrial translation.

Here, we report novel biallelic NSUN3 missense variants in a South Asian patient with early-onset mitochondrial encephalomyopathy and seizures.

\section{Patients and Methods}

\section{Patient Cohort}

A cohort comprising 167 South Asian patients with suspected primary mitochondrial disease (PMD), based on clinical, pathological and biochemical findings, was included. Forty-eight patients had early-onset mitochondrial encephalomyopathy. Informed written consent for diagnostic and research studies was obtained from all subjects. The study was approved by the Institutional Ethical Committee (IEC) of CSIR-Centre for 
Cellular and Molecular Biology, Hyderabad, India, and Nizam's Institute of Medical Sciences (NIMS), Hyderabad, India.

\section{Mitochondrial DNA Sequencing}

DNA was extracted from blood leucocytes using a standard phenol-chloroform method (Thangaraj et al. 2002), with minor modifications. Complete mtDNA was amplified using 24 sets of primers to generate overlapping amplicons, purified and directionally sequenced using BigDye terminator cycle sequencing kit and ABI3730 XL Genetic Analyzer (Rieder et al. 1998).

\section{Analysis of Mitochondrial-Related Nuclear Genes}

All mitochondrial nuclear genes ( $P O L G, P O L G 2, T K 2$, SLC25A4, DGUOK, MPV17, RRM2B, MFN2, SPG7, $A F G 3 L 2, R N A S E H 1$ and NSUN3) were analysed using custom-designed primers (http://frodo.wi.mit.edu) and bidirectionally Sanger sequenced using BigDye terminator cycle sequencing kit and 3730XL Genetic Analyzer. DNA variations were identified after assembling each patient's sequence with the reference sequence using Auto-assembler software (Applied Bio-systems).

\section{In Silico Functional Analysis}

To evaluate the potential functional impact of the identified missense variants, we utilised a variety of pathogenicity prediction programs, including SIFT (http://sift.jcvi.org/), PolyPhen-2 (http://genetics.bwh.harvard.edu/pph2//) and MutationTaster (http://www.mutationtaster.org/).

\section{Results}

The novel compound heterozygous NSUN3 missense variants c. $421 \mathrm{G}>\mathrm{C}$ (p.A141P) and c.454T $>$ A (p.C152S) were detected in one patient with early-onset mitochondrial encephalomyopathy and seizures (Fig. 1a, II:3). The proband, an 8-monthold boy born to consanguineous parents following a normal pregnancy, presented at 4 months of age with lactic acidosis, global developmental delay, hypotonia, muscle weakness and seizures. There was a family history of lactic acidosis in the proband's elder sister (Fig. 1a, II:1). Brain magnetic resonance imaging (MRI) revealed cerebral atrophy and white matter hyperintensities involving both cerebral hemispheres, particularly the frontal and temporal lobes (Fig. 1b). Segregation studies confirmed that the proband's unaffected father (Fig. 1a, I:1) and elder brother (Fig. 1a, II:2) were carriers of the c.454T>A (p.C152S) variant, while the proband's unaffected

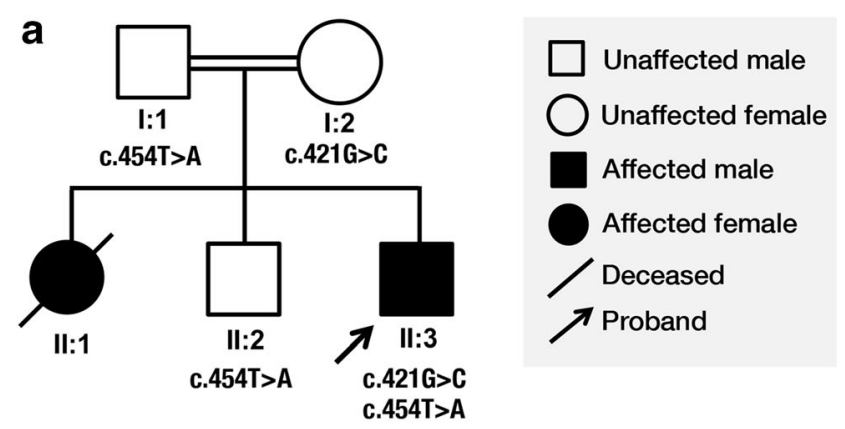

b

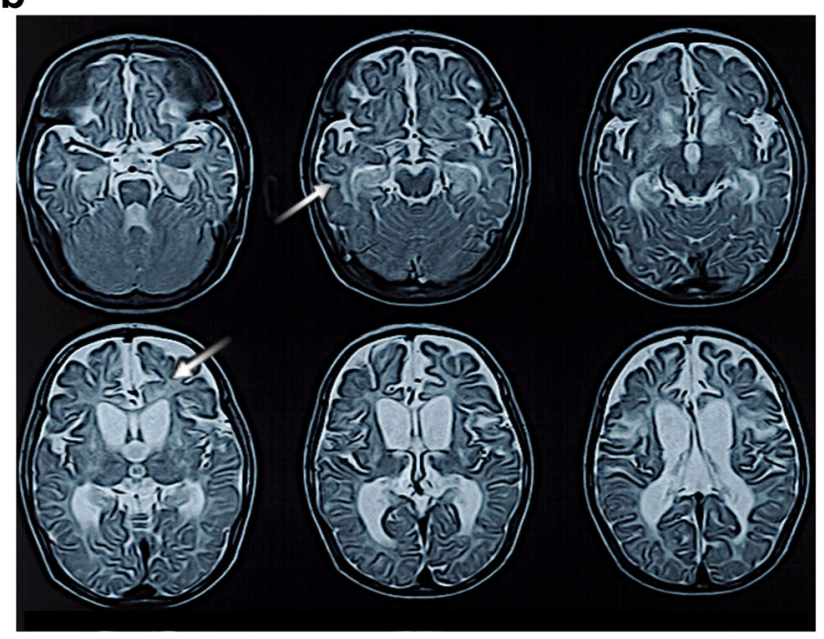

Fig. 1 Pedigree demonstrating segregation of the novel NSUN3 variants (a). Proband's unaffected father (I:1). Proband's unaffected mother (I:2). Proband's elder sister (II:1). Proband's elder brother (II:2). Proband (II:3). T2-weighted axial images demonstrate white matter hyperintensities in both cerebral hemispheres, predominantly affecting the frontal and temporal lobes, arrows (b)

mother (Fig. 1a, I:2) was a carrier for the c. $421 \mathrm{G}>\mathrm{C}$ (p.A141P) variant.

Both variants reside in exon 3 of the NSUN3 gene (Fig. 2 a and $b$ ), within a highly conserved region of the protein (Fig. 2c), and are absent in the 1000 Genomes Project (http://www. 1000genomes.org/about), Exome Aggregation Consortium (ExAC, http://exac.broadinstitute.org/), Genome Aggregation Database (gnomAD, http://gnomad-old. broadinstitute.org/) and 485 ethnically matched healthy control subjects. In silico functional analysis confirmed that the variants have a high probability of being pathogenic (Table 1). Unfortunately, muscle tissue and cultured fibroblasts were unavailable for biochemical or functional studies.

\section{Discussion}

We report a South Asian patient with early-onset mitochondrial encephalomyopathy and seizures harbouring novel, compound heterozygous NSUN3 missense variants c.421G >C (p.A141P) and c.454T>A (p.C152S). NSUN3 
Fig. 2 Schematic diagram of the NSUN3 gene with previously reported variants (red letters) and the novel variants identified in this study (black letters) annotated (a). Sequence analysis of NSUN3 in the proband confirming the c. $421 \mathrm{G}>\mathrm{C}$ (p.A141P) and c.454T $>$ A (p.C152S) NSUN3 variants, arrows (b). Evolutionary conservation data for NSun3 amino acid sequence across species at positions 141 and 152 , arrows (c)

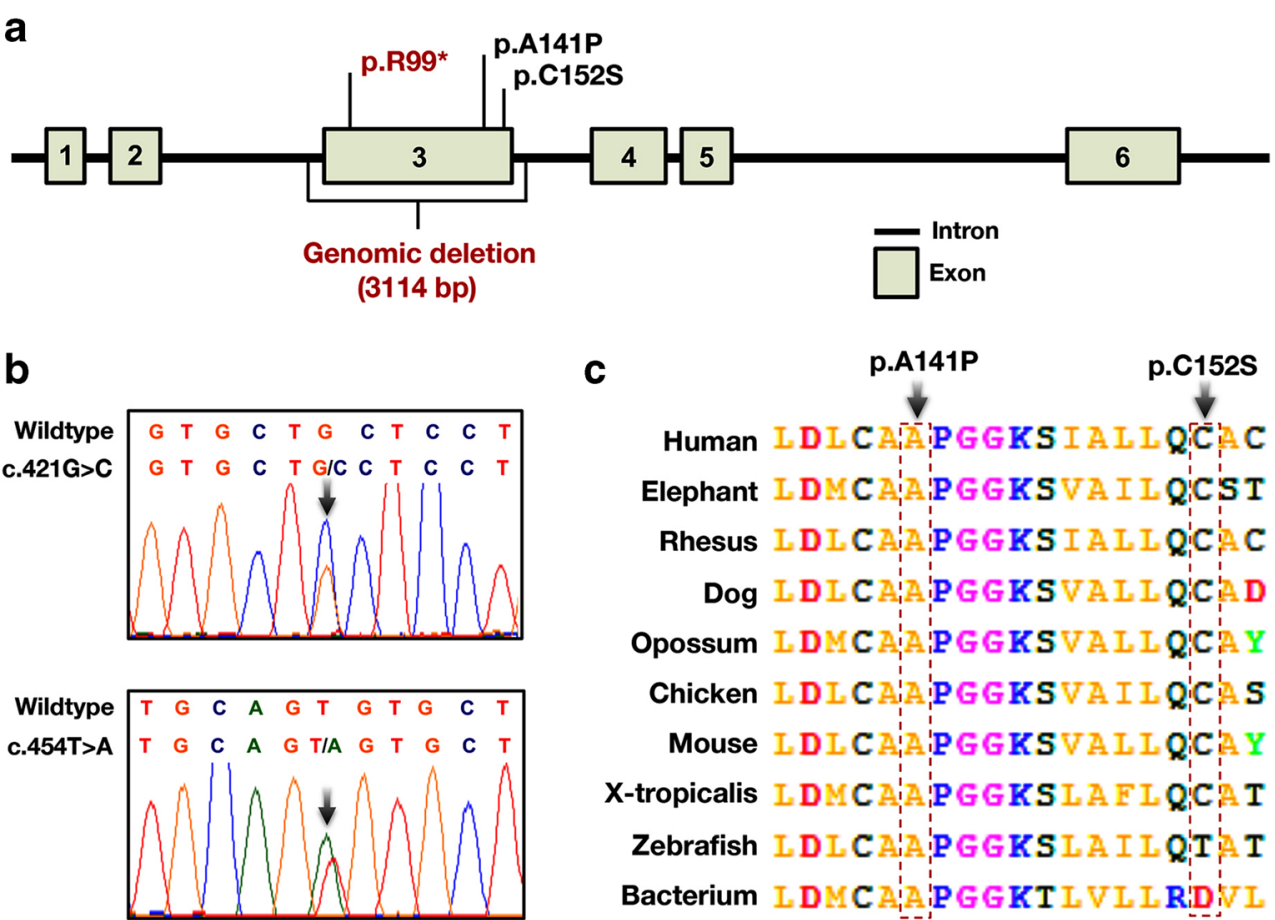

comprises six exons that encode a 340 amino acid protein. The c. $421 \mathrm{G}>\mathrm{C}$ and c. $454 \mathrm{~T}>\mathrm{A}$ missense variants reside within exon 3 and result in substitutions of an alanine to proline (p.A141P) and a cysteine to serine (p.C152S), respectively. The two previously reported pathogenic NSUN3 variants (c.123-615_466+2155del and c.295C>T) also occur in exon 3 (Van Haute et al. 2016). Thus, this region of the gene potentially represents a hotspot for NSUN3-related mitochondrial disease.

NSun3 is a novel human m5C RNA methyltransferase that specifically methylates mitochondrial tRNA ${ }^{\text {Met }}$. NSUN3-knockout cells demonstrate reduced mitochondrial protein synthesis and oxygen consumption resulting in decreased mitochondrial activity (Trixl et al. 2018). Disease-associated mt-tRNA ${ }^{\text {Met }}$ point mutations that impair Nsun3-mediated methylation have pathological consequences (Nakano et al. 2016), and loss of function mutations in $N S U N 3$ are reported to cause severe multisystem mitochondrial disease associated with combined OXPHOS deficiency (Van Haute et al. 2016). In the current study, we expand the molecular and clinical spectrum of NSUN3-related mitochondrial disease to include two novel NSUN3 missense variants and seizures.

The following lines of evidence support the pathogenic effects of the c.421G $>$ C (p.A141P) and c.454T $>$ A (p.C152S) NSUN3 variants: (1) conservation data suggests p.A141 and p.C152 are highly conserved amino acid residues of the Nsun3 protein; (2) both variants are absent from the 1000 Genomes Project, ExAC, gnomAD and 485 ethnically matched healthy control subjects; (3) segregation studies confirm the variants are trans-acting in the proband, while both parents and unaffected sibling are heterozygous carriers; (4) in silico functional analyses

Table 1 Summary of the in silico functional analysis, open access genetic databases and in-house ethnically matched healthy control subjects used to evaluate the reported NSUN3 variants

\begin{tabular}{|c|c|c|c|c|c|c|c|c|c|c|}
\hline Mutation & $\begin{array}{l}\text { Amino acid } \\
\text { change }\end{array}$ & SIFT & Polyphen-2 & $\begin{array}{l}\text { Mutation } \\
\text { Taster }\end{array}$ & ExAC & EVS & $\begin{array}{l}1000 \\
\text { Genomes }\end{array}$ & HGVD & gnomAD & $\begin{array}{l}{ }^{*} \text { Controls } \\
n=485\end{array}$ \\
\hline c. $421 \mathrm{G}>\mathrm{C}$ & p.A141P & $\begin{array}{l}\text { Deleterious } \\
\quad(\text { score } 0.00)\end{array}$ & $\begin{array}{l}\text { Probably } \\
\text { Damaging }\end{array}$ & Disease causing & No & No & No & No & No & No \\
\hline c. $454 \mathrm{~T}>\mathrm{A}$ & p.C152S & $\begin{array}{l}\text { Tolerated } \\
\quad(\text { score } 0.50)\end{array}$ & $\begin{array}{l}\text { Possibly } \\
\text { Damaging }\end{array}$ & Disease causing & No & No & No & No & No & No \\
\hline
\end{tabular}

ExAC Exome Aggregation Consortium, EVS Exome Variant Server, HGVD Human Genetic Variation Database

*Ethnically matched healthy control subjects 
predict the variants to be pathogenic with high probability scores; and (5) no known pathogenic variants were detected in either mtDNA or disease-associated mitochondrial nuclear maintenance genes.

In conclusion, we report novel biallelic NSUN3 missense variants causing early-onset mitochondrial encephalomyopathy and seizures, thereby expanding the molecular and phenotypic spectrum of NSUN3-related mitochondrial disease.

Acknowledgements We sincerely thank the patient and their family for their participation in this study and to whom our research is dedicated.

Funding information AP was supported by the Science and Engineering Research Board (SERB) (EEQ/2019/000411), KT has been supported by the research funding (GAP0517) and J C Bose Fellowship from SERB, DST, Government of India. RDSP is supported by a Medical Research Council Clinician Scientist Fellowship (MR/S002065/1).

\section{Compliance with Ethical Standards}

Informed written consent for diagnostic and research studies was obtained from all subjects. The study was approved by the Institutional Ethical Committee (IEC) of CSIR-Centre for Cellular and Molecular Biology, Hyderabad, India, and Nizam's Institute of Medical Sciences (NIMS), Hyderabad, India.

Competing Interests The authors declare that they have no conflicts of interest.

Open Access This article is licensed under a Creative Commons Attribution 4.0 International License, which permits use, sharing, adaptation, distribution and reproduction in any medium or format, as long as you give appropriate credit to the original author(s) and the source, provide a link to the Creative Commons licence, and indicate if changes were made. The images or other third party material in this article are included in the article's Creative Commons licence, unless indicated otherwise in a credit line to the material. If material is not included in the article's Creative Commons licence and your intended use is not permitted by statutory regulation or exceeds the permitted use, you will need to obtain permission directly from the copyright holder. To view a copy of this licence, visit http://creativecommons.org/licenses/by/4.0/.

\section{References}

Boccaletto P, Machnicka MA, Purta E, Piatkowski P, Baginski B, Wirecki TK, de Crecy-Lagard V, Ross R, Limbach PA, Kotter A et al (2018) MODOMICS: a database of RNA modification pathways. 2017 update. Nucleic Acids Res 46:D303-D307

Haag S, Sloan KE, Ranjan N, Warda AS, Kretschmer J, Blessing C, Hübner B, Seikowski J, Dennerlein S, Rehling P, Rodnina MV, Höbartner C, Bohnsack MT (2016) NSUN3 and ABH1 modify the wobble position of mt-tRNAMet to expand codon recognition in mitochondrial translation. EMBO J 35:2104-2119

Hsu PJ, Shi H, He C (2017) Epitranscriptomic influences on development and disease. Genome Biol 18:197

Hussain S, Aleksic J, Blanco S, Dietmann S, Frye M (2016) Characterizing 5 -methylcytosine in the mammalian epitranscriptome. Genome Bio $14: 215$

Machnicka MA, Milanowska K, Osman Oglou O, Purta E, Kurkowska M, Olchowik A (2013) MODOMICS: a database of RNA modification pathways-2013 update. Nucleic Acids Res 41:D262-D267

Nakano S, Suzuki T, Kawarada L, Iwata H, Asano K, Suzuki T (2016) NSUN3 methylase initiates 5-formylcytidine biogenesis in human mitochondrial tRNA (Met). Nat Chem Biol 12:546-551

Rieder MJ, Taylor SL, Tobe VO, Nickerson DA (1998) Automating the identification of DNA variations using quality-based fluorescence re-sequencing: analysis of the human mitochondrial genome. Nucleic Acids Res 26:967-973

Rozov A, Demeshkina N, Khusainov I, Westhof E, Yusupov M, Yusupova G (2016) Novel base-pairing interactions at the tRNA wobble position crucial for accurate reading of the genetic code. Nat Commun 7:10457

Thangaraj K, Joshi MB, Reddy AG, Gupta NJ, Chakravarty B, Singh L (2002) CAG repeat expansion in the androgen receptor gene is not associated with male infertility in Indian populations. J Androl 23: $815-818$

Trixl L, Amort T, Wille A, Zinni M, Ebner S, Hechenberger C, Eichin F, Gabriel H, Schoberleitner I, Huang A, Piatti P, Nat R, Troppmair J, Lusser A (2018) RNA cytosine methyltransferase Nsun3 regulates embryonic stem cell differentiation by promoting mitochondrial activity. Cell Mol Life Sci 75:1483-1497

Van Haute L, Dietmann S, Kremer L, Hussain S et al (2016) Deficient methylation and formylation of mt-tRNA (Met) wobble cytosine in a patient carrying mutations in NSUN3. Nat Commun 7:12039

Publisher's Note Springer Nature remains neutral with regard to jurisdictional claims in published maps and institutional affiliations. 